\title{
DIAGNÓSTICO HIDROQUÍMICO DAS ÁGUAS SUBTERRÂNEAS DE QUISSAMÃ - RJ
}

\author{
Priscila Alves Marques Fernandes \\ Mestre em Engenharia Civil/ UENF \\ priscilaamf@yahoo.com.br \\ Maria da Glória Alves \\ Doutora em Geologia/ UENF \\ mgalvesuenf@gmail.com \\ José Luiz Ernandes Dias Filho \\ Mestre em Engenharia Civil/ UENF \\ jlernandes@hotmail.com \\ Gerson Cardoso da Silva Júnior \\ Doutor em Hridrogeologia/ UFRJ \\ gerson@acd.ufrj.br.
}

Recebido: 13 de julho de 2013. Revisado: 02 de agosto de 2013. Aceito: 03 de setembro de 2013. Publicado online: 04 de setembro de 2013.

\section{RESUMO}

O município de Quissamã localiza-se na Microrregião de Macaé/RJ, emancipado em 1989. Tem como principais atividades econômicas criação de bovinos, cultura da cana de açúcar, coco verde e abacaxi. O desenvolvimento ocorreu de forma acelerada e desordenada após sua emancipação, gerando maior demanda de água e ocasionando problemas no abastecimento. Objetiva-se avaliar a hidroquímica das águas subterrâneas do município. Foi criada base de dados georreferenciados para elaboração de mapas, realizadas análises hidroquímicas em 09 pontos e utilizados os diagramas de Piper e de Stiff para classificação das águas. Os resultados mostraram que as águas subterrâneas indicaram presença de elementos fora do padrão: $\mathrm{Al}, \mathrm{Fe}, \mathrm{NO}_{2}, \mathrm{~Pb}, \mathrm{Hg}$, Se, Na. De acordo com os diagramas de Piper e de Stiff os poços profundos e rasos de Quissamã possuem predominância em sua classificação de águas cloretadas sódicas. Conclui-se que a água subterrânea quando captada de forma adequada apresenta baixo custo de tratamento.

Palavras-chave: águas subterrâneas; análises hidroquímicas; diagramas de Piper e de Stiff; Quissamã.

Persp. online: exatas \& eng., Campos dos Goytacazes, 3 (6), 25-41, 2013

seer.perspectivasonline.com.br 


\begin{abstract}
The municipality is located in Quissamã Microregion Macaé/RJ, emancipated in 1989. Its main economic activities in cattle, cultivation of sugar cane, coconut and pineapple. The development occurred in an accelerated and disorderly after his emancipation, creating greater demand for water supply and causing problems. The objective is to assess the hydrochemistry of groundwater in the city. It was created georeferenced database to produce maps, hydrochemical analyzes in 09 points and used the Piper and Stiff diagrams for classification of waters. The results showed that the groundwater indicated the presence of nonstandard elements: $\mathrm{Al}, \mathrm{Fe}, \mathrm{NO}_{2}, \mathrm{~Pb}, \mathrm{Hg}$, Se, Na. According to Piper and Stiff diagrams of the deep wells and shallow Quissamã have predominance in its classification of chlorinated water with sodium. It follows that the ground water when captured appropriately has a low cost of treatment.
\end{abstract}

Keywords: groundwater, hydrochemical analyzes; Piper diagrams and Stiff; Quissamã.

\title{
1. INTRODUÇÃO
}

A água é reconhecida pela ciência como o ambiente em que surgiu a própria vida e também como solvente universal. A vida não existe sem água líquida.

O uso de águas subterrâneas tem aumentado significativamente nas últimas décadas. Particularmente nas áreas rurais dos países em desenvolvimento, em regiões áridas e semi-áridas e nas ilhas. Em algumas cidades é a principal fonte de abastecimento de água. A agricultura e sistemas de irrigação em particular, em muitas partes do mundo, dependem fortemente dos recursos hídricos subterrâneos (UNESCO, 2007).

No entanto, o controle gerencial sobre o desenvolvimento dos recursos hídricos e da sua proteção é muitas vezes deficiente, o que leva à exploração descontrolada e contaminação dos aquíferos. A solução está intimamente ligada ao planejamento e a gestão da água sendo que o principal objetivo deste processo é assegurar a quantidade, qualidade, segurança e sustentabilidade das águas subterrâneas (Freeze e Cherry, 1979).

Os padrões da U.S Enviromental Protection Agency (USEPA, 1975), World Health Organization European Standard (OMS, 1970) recomendam concentrações limites aceitáveis para consumo humano. Freeze e Cherry (1979) e Fetter (2001) descrevem os constituintes químicos das águas subterrâneas, contaminação e sua qualidade, mostrando que o modelo principal de uso é para o consumo humano; (Santos, 1997) apresenta valores permitidos para águas, dentro do padrão do uso estabelecido. No Brasil, alguns parâmetros físico-químicos são adotados para avaliação da qualidade da água para consumo humano e estão relacionadas a Portaria $n^{0}$ 518/2004 (Brasil, 2004) e Portaria no 2914/ 2011 (Brasil, 2012), além de serem recomendados pela Organização Mundial da Saúde (OMS, 2004), no que tange à potabilidade da água.

Do ponto de vista hidrogeológico a qualidade é tão importante quanto à quantidade, a disponibilidade dos recursos hídricos para determinados usos depende fundamentalmente da qualidade físico-química, biológica e radiológica das águas (Santos, 1997).

Persp. online: exatas \& eng., Campos dos Goytacazes, 3 (6), 25-41, 2013

seer.perspectivasonline.com.br 


\subsection{Caracterização da Área de Estudo}

O estudo foi desenvolvido no Município de Quissamã, pertencente à Microrregião de Macaé e à Região Norte Fluminense do Estado do Rio de Janeiro, possui $715,88 \mathrm{~km}^{2}$ de território, banhado pelo Oceano Atlântico ao longo de aproximadamente $45,00 \mathrm{~km}$ de extensão de costa e uma população de 18.315 (dezoito mil trezentos e quinze) habitantes (IBGE, 2010).

O município de Quissamã faz divisa ao norte com Campos dos Goytacazes, ao sul com o Oceano Atlântico, a noroeste com Conceição de Macabu e a leste com Carapebus (Figura 1).

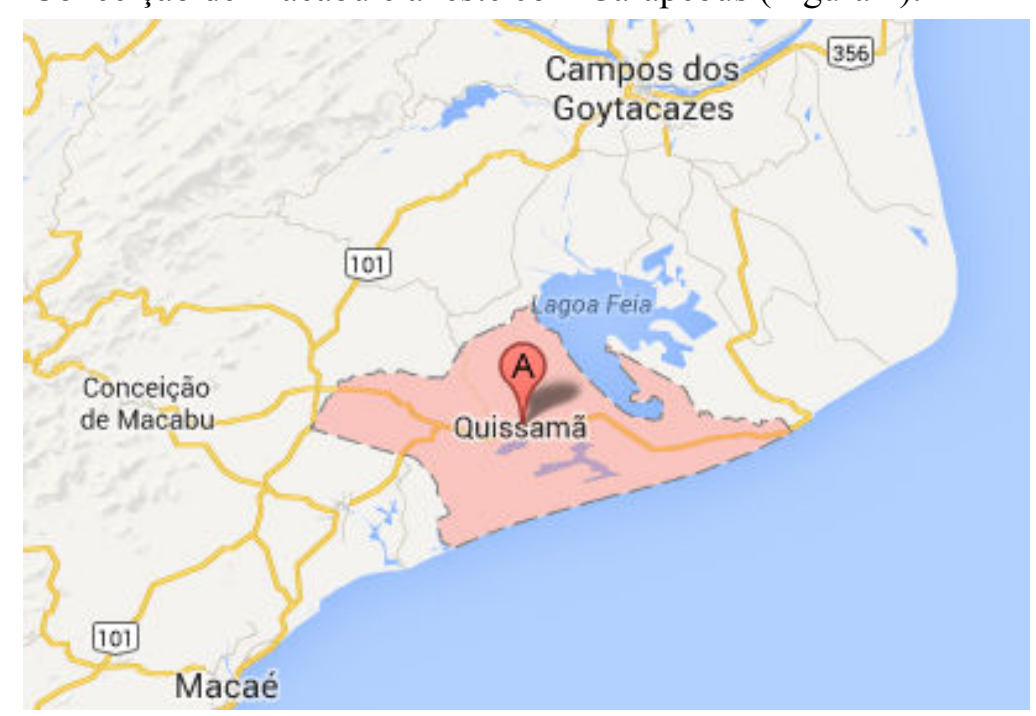

Figura 1 - Localização da área de estudo (GOOGLE, 2013)

\subsection{Abastecimento de água}

Devido ao rápido desenvolvimento econômico que a região de Quissamã vem apresentando nos últimos anos, provocado por sua emancipação, ocorre uma maior demanda de abastecimento de água, tanto para a indústria e agricultura quanto para o consumo da população devido à instalação de novos pólos industriais e da ampliação de práticas agrícolas.

A maior parte do abastecimento de água de Quissamã é realizada pela Companhia Estadual de Águas e Esgotos do Rio de Janeiro (CEDAE), que capta água da Lagoa Feia e abastece a população da região.

Ainda existe a utilização de carros-pipa, nascentes e o consumo de água de poços particulares, rasos (cacimba) e profundos, onde em certos locais são utilizados como única fonte alternativa de abastecimento. Estes poços do tipo cacimba, que a população perfura na cidade de Quissamã, são vulneráveis a diversos tipos de poluição e/ou contaminação, como: "fossas sépticas", tubulações de esgoto com fissuras, disposição inadequada de resíduos sólidos, cemitérios, criação de animais, atividades agrícolas, entre outras.

O objetivo da pesquisa foi realizar uma avaliação dos recursos hídricos subterrâneos do município de Quissamã, pois este convive com constante escassez de abastecimento de água. 


\subsection{Qualidade da Água Subterrânea}

A qualidade da águas subterrâneas se dá pela dissolução dos minerais presentes nas rochas que constituem os aqüíferos por ela percolados, podendo ter influência de outros fatores como composição da água de recarga, tempo de contato água/meio físico, clima e até mesmo a poluição causada pelas atividades humanas (CAPUCCI ET AL, 2001).

Segundo Santos (2000) a qualidade da água refere-se a um padrão semelhante ao encontrado nos rios e nascentes antes da ação do homem. O grau de pureza desejável depende do uso a que se destine e assim devem ser consideradas as propriedades das águas.

Enquadram-se nas características das propriedades físicas os seguintes aspectos: temperatura, cor, odor, sabor, turbidez e sólidos em suspensão.

Dentre as propriedades iônicas consideram-se: condutividade elétrica, dureza, alcalinidade, $\mathrm{pH}$, sólidos totais dissolvidos, demanda química de oxigênio e demanda bioquímica de oxigênio.

As propriedades biológicas são analisadas sob o ponto de vista de organismos patogênicos que não existem na água em condições ditas naturais. Esses seres são normalmente habitantes do próprio organismo humano e são eliminados por fezes, veículo dos seres patogênicos para a água (TODD, 1967).

\subsubsection{Hidroquímica}

$\mathrm{O}$ estudo hidroquímico tem por finalidade identificar e quantificar as principais propriedades e constituintes das águas, enquanto em correlação com a geoquímica pode-se relacionar estes parâmetros ao meio físico no qual ela se encontra. Esta avaliação terá como enfoque sempre o uso para o qual se destina a água analisada; por vezes este foco é perdido em benefício de uma ponderação que se preocupa apenas com os aspectos quantitativos do aqǘfero, em detrimento do respeito às suas especificidades químicas (CUSTÓDIO E LAMAS, 1983).

O conjunto de todos os elementos que compõem a água permite estabelecer padrões de qualidade, classificando-a, assim, de acordo com seus limites estudados e seus usos para consumo humano ou agrícola, entre outros (Tabela 1).

Tabela 1 - Efeitos dos metais tóxicos no ser humano, ingeridos através da água (Teixeira, 1998)

\begin{tabular}{|l|l|l|}
\hline Elementos & \multicolumn{1}{|c|}{ Ingestão } & \multicolumn{1}{c|}{ Conseqüências } \\
\hline Alumínio & $\begin{array}{l}\text { Ingerido através da água é absorvido pelo } \\
\text { aparelho digestivo e todo o excesso é } \\
\text { evacuado pelas fezes. }\end{array}$ & $\begin{array}{l}\text { O acúmulo de alumínio no corpo poderá danificar o sistema } \\
\text { nervoso central e a estrutura óssea do ser humano. Não } \\
\text { comprovado a sua ligação com a doença de Mal de } \\
\text { Alzheimer. }\end{array}$ \\
\hline Cádmio & $\begin{array}{l}\text { O metabolismo ocorre pela absorção } \\
\text { gastrointestinal, penetrando na circulação } \\
\text { sanguínea e concentrando no plasma, } \\
\text { alcançando os glóbulos vermelhos, depois } \\
\text { segue para os rins, fígado, pâncreas e } \\
\text { glândulas salivares (tireóide). }\end{array}$ & $\begin{array}{l}\text { A ingestão de água contaminada por cádmio é caracterizada } \\
\text { por sintomas reumáticos com intensa dor nos ossos, } \\
\text { provocada pela perda de minerais dos ossos, fazendo com } \\
\text { que fiquem extremamente flexíveis. }\end{array}$ \\
\hline
\end{tabular}

Persp. online: exatas \& eng., Campos dos Goytacazes, 3 (6), 25-41, 2013

seer.perspectivasonline.com.br 


\begin{tabular}{|c|c|c|}
\hline Chumbo & $\begin{array}{l}\text { Se ingerido através da água, pode provoca } \\
\text { reações com outros elementos. }\end{array}$ & $\begin{array}{l}\text { Em altas concentrações é capaz de provocar lesões no } \\
\text { sistema neuromuscular e problemas na circulação, no } \\
\text { cérebro e no tubo digestivo, apresentando sintomas como } \\
\text { anorexia, náuseas, paralisia, distúrbios visuais, anemia e } \\
\text { convulsões. }\end{array}$ \\
\hline Cobre & $\begin{array}{l}\text { Normalmente, é encontrado nos tecidos } \\
\text { dos organismos dos animais e vegetais, } \\
\text { sendo benéfico para o homem em } \\
\text { pequenas concentrações. Sua deficiência } \\
\text { em criança pode causar anemia nutricional. }\end{array}$ & $\begin{array}{l}\text { O cobre não é considerado elemento tóxico, mas em altas } \\
\text { concentrações podem produzir vômitos e ocasionar lesões ao } \\
\text { fígado. }\end{array}$ \\
\hline Cromo & $\begin{array}{l}\text { Pode ser encontrado na maioria dos } \\
\text { sistemas biológicos, sendo ainda } \\
\text { desconhecidos seus efeitos benéficos ao } \\
\text { ser humano. }\end{array}$ & $\begin{array}{l}\text { O ácido crômico, os cromatos e bicromatos, provocam } \\
\text { problemas à saúde quando inalados, ou por contato com a } \\
\text { pele. }\end{array}$ \\
\hline Mercúrio & & $\begin{array}{l}\text { A intoxicação crônica com os derivados orgânicos do } \\
\text { mercúrio normalmente está associada a acidentes ou } \\
\text { contaminações localizadas no ambiente. Seus efeitos tóxicos } \\
\text { a saúde humana, podem provocar náuseas, vômitos, cólicas } \\
\text { abdominais, diarréia sangüínea, danos aos rins, e poderá ser } \\
\text { letal em altas concentrações. }\end{array}$ \\
\hline Zinco & $\begin{array}{l}\text { É um metal benéfico ao ser humano em } \\
\text { baixas concentrações, atuando nas } \\
\text { atividades da insulina, sendo que diversos } \\
\text { compostos dependem da sua presença para } \\
\text { seu metabolismo. }\end{array}$ & $\begin{array}{l}\text { Em altas concentrações pode ser acumular no fígado, } \\
\text { pâncreas, próstata, sulco pancreático e líquido seminal. } \\
\text { Alguns sais de zinco como, o sulfato de zinco são tóxicos, } \\
\text { mas não é conhecida sua dose letal. }\end{array}$ \\
\hline Ferro & $\begin{array}{l}\text { É o quarto elemento mais abundante na } \\
\text { crosta terrestre. }\end{array}$ & $\begin{array}{l}\text { Sua carência causa anemia e seu excesso aumenta a } \\
\text { incidência de problemas cardíacos e diabetes. Porém, a sua } \\
\text { inalação de ferro ou de seus óxidos produz pneumoconiose, } \\
\text { causando bronquite crônica. }\end{array}$ \\
\hline
\end{tabular}

\subsubsection{Princípios de Classificação das Águas- Técnicas Gráficas}

a) Diagrama Triangular de Piper - O diagrama de Piper (Figura 2) é utilizado para classificar os distintos grupos de águas quanto aos íons dominantes (cloretada, sódica, carbonatada, magnesiana e outras). Este sistema de classificação é baseado na sobrepujança de determinados íons sobre outros. A água é classificada quanto ao ânion ou cátion, cuja concentração, expressa em $\mathrm{m}_{\mathrm{eq}} / \mathrm{L}$, ultrapassa em $50 \%$ suas respectivas somas. Se nenhum deles ultrapassa este valor, a água é denominada de acordo com os dois ânios ou cátions mais abundantes. Para plotar no diagrama, transforma-se separadamente o valor de cada íon expresso em $\mathrm{m}_{\mathrm{eq}} / \mathrm{L}$ em percentagem dos totais de ânions e de cátions (SANTOS, 2000); 


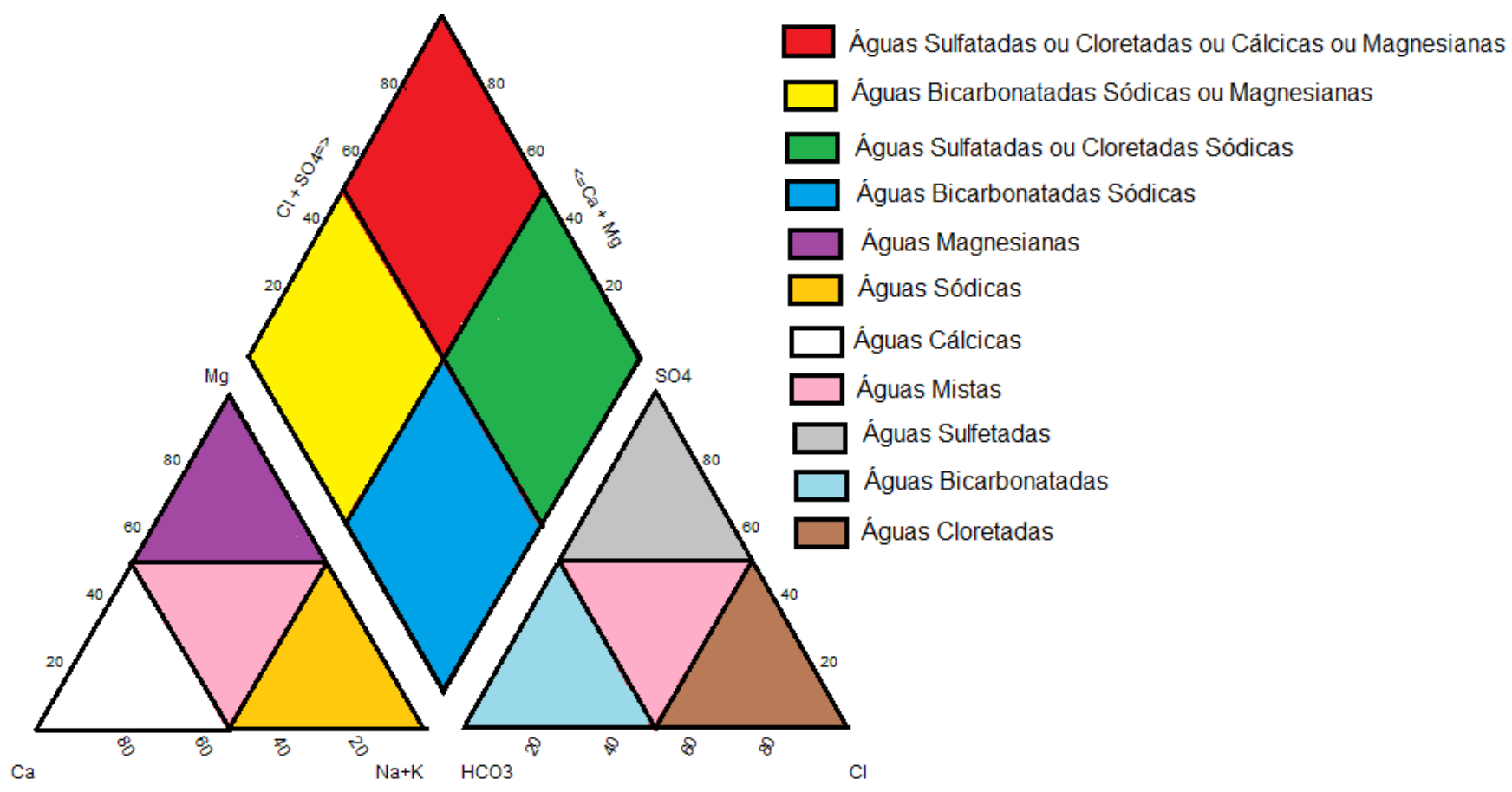

Figura 2 - Diagrama de Piper

b) Diagrama de Stiff - Todas as concentrações iônicas em $\mathrm{m}_{\mathrm{eq}} / \mathrm{L}$ ou $\% \mathrm{~m}_{\mathrm{eq}} / \mathrm{L}$ são representadas sobre linhas paralelas horizontais. Ligando-se todos os pontos respectivos, obtém-se a figura geométrica característica da água analisada.

\section{METODOLOGIA}

\subsection{Levantamento de Campo}

As campanhas de campo realizadas durante o ano de 2012 possuíram como objetivo promover a criação de uma base de dados para uma melhor compreensão e avaliação das águas subterrâneas da região. Nessas campanhas foram medidos em campo: temperatura, $\mathrm{pH}$ e condutividade elétrica (CE) e coletadas amostras para análise de parâmetros hidroquímicos.

O mapa de geologia da região em estudo (Figura 3) foi escolhido para orientar o trabalho de coleta das amostras de água no campo, pois a qualidade da água reflete os meios por onde ela percola. Os poços rasos e profundos coletados estão localizados em núcleos urbanos e em áreas rurais.

A Tabela 2 apresenta a identificação do poço, as coordenadas UTM e localidade dos pontos de coleta para a realização das análises hidroquímicas e a Tabela 3 os parâmetros físicos medidos no campo destes pontos.

Persp. online: exatas \& eng., Campos dos Goytacazes, 3 (6), 25-41, 2013 
Tabela 2 - Cadastro dos pontos de coleta para a as análises hidroquímicas

\begin{tabular}{|c|c|c|c|c|}
\hline \multicolumn{2}{|c|}{$\begin{array}{c}\text { Identificação } \\
\text { do Poço }\end{array}$} & $\mathrm{E}$ & $\mathrm{N}$ & Localidade \\
\hline \multirow{4}{*}{$\begin{array}{l}0 \\
0 \\
0 \\
\equiv \\
0 \\
0 \\
0 \\
0 \\
0 \\
0 \\
0 \\
0\end{array}$} & 1 & 0242679 & 7555852 & Fazenda Vista Alegre 1 \\
\hline & 2 & 0232644 & 7553796 & Morro Alto \\
\hline & 3 & 0246817 & 7561440 & Fazenda Machadinha \\
\hline & 4 & 0242713 & 7556023 & Fazenda Vista Alegre 2 \\
\hline \multirow{5}{*}{ 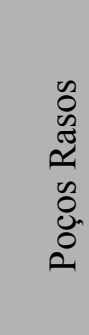 } & 5 & 0242340 & 7557055 & Santa Catarina \\
\hline & 6 & 0241132 & 7553414 & São José \\
\hline & 7 & 0251159 & 7554078 & Fazenda Pitanga \\
\hline & 8 & 0276516 & 7553361 & São Miguel \\
\hline & 9 & 0249026 & 7557318 & Conde de Araruama \\
\hline
\end{tabular}
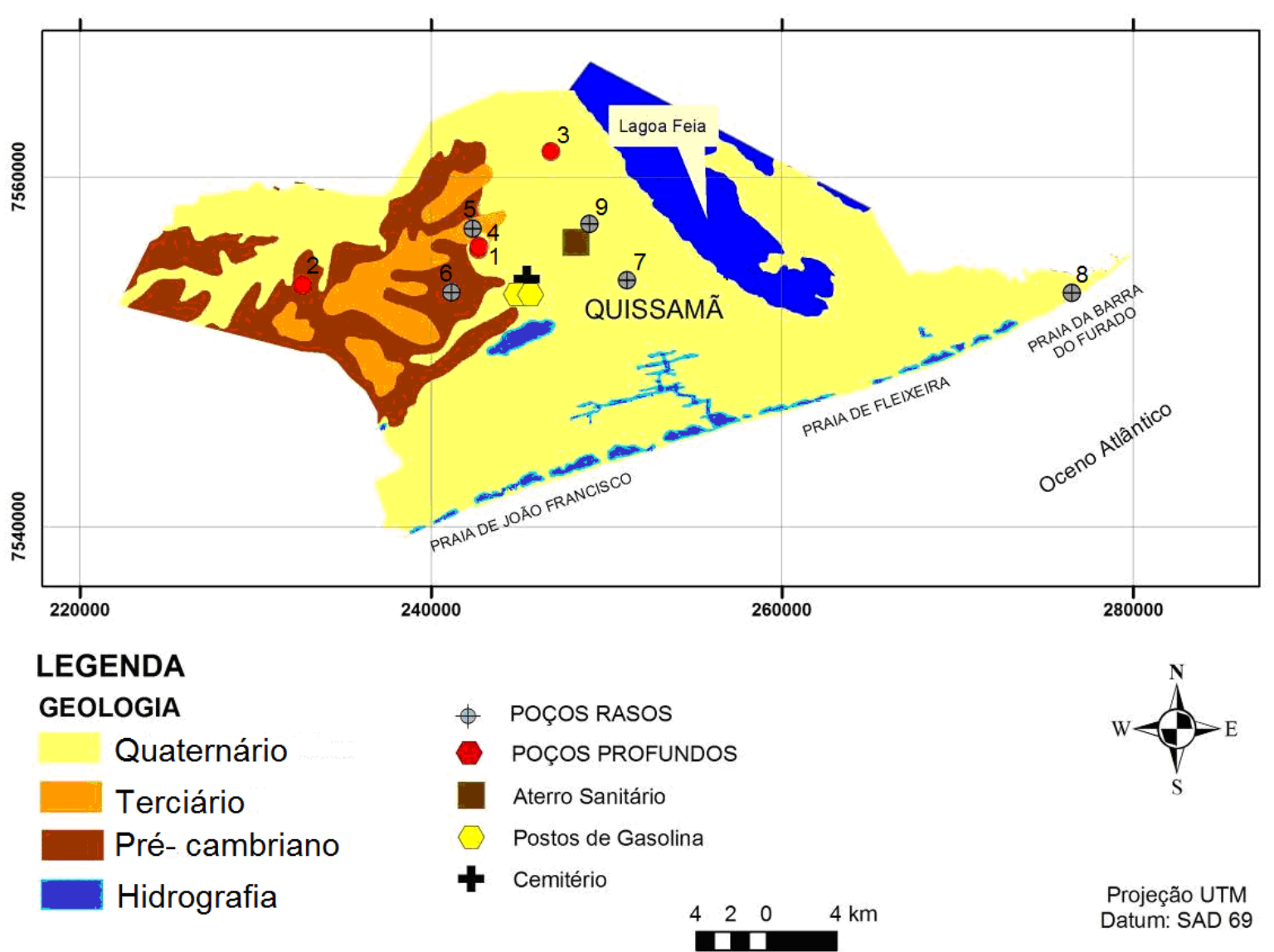

Projeção UTM Datum: SAD 69

Figura 3 - Mapa de espacialização dos pontos de coleta para a realização das análises hidroquímicas (Fonte: Oficina de geologia e geoprocessamento - OFIGEO/UENF. Compilado do CPRM, 2001) 
Tabela 3 - Parâmetros físicos medidos no campo para a realização das análises hidroquímicas

\begin{tabular}{|c|c|c|c|c|c|c|}
\hline \multicolumn{2}{|c|}{$\begin{array}{c}\text { Identificação } \\
\text { do Poço }\end{array}$} & $\begin{array}{c}\text { Nível d'água } \\
\text { (m) }\end{array}$ & $\begin{array}{l}\text { Profundidade } \\
\text { (m) }\end{array}$ & $\mathrm{pH}$ & $\begin{array}{c}\text { Temperatura } \\
\left({ }^{\circ} \mathrm{C}\right) *\end{array}$ & $\begin{array}{l}\text { Cond. Elét } \\
(\mu \mathrm{s} / \mathrm{cm})^{*}\end{array}$ \\
\hline \multirow{4}{*}{$\begin{array}{l}n \\
0 \\
0 \\
\equiv \\
0 \\
0 \\
0 \\
0 \\
0 \\
0 \\
0 \\
0\end{array}$} & 1 & 29,24 & 36,00 & 4,1 & 28,7 & 900 \\
\hline & 2 & Poço lacrado & 101,00 & 5,1 & 29,6 & 609 \\
\hline & 3 & 119,44 & 128,00 & 5,4 & 28,3 & 3240 \\
\hline & 4 & 16,68 & 30,00 & 5,0 & 26,6 & 441 \\
\hline \multirow{5}{*}{ 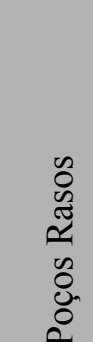 } & 5 & 3,00 & 4,00 & 3,6 & 29,2 & 192 \\
\hline & 6 & 8,00 & 13,00 & 5,0 & 26,3 & 261 \\
\hline & 7 & 2,05 & - & 6,7 & 29,1 & 146 \\
\hline & 8 & 0,62 & - & 7,0 & 28,3 & 147 \\
\hline & 9 & 1,86 & 10,00 & 6,6 & 26,6 & 368 \\
\hline
\end{tabular}

\begin{tabular}{|c|c|c|}
\hline $\begin{array}{c}\text { Valores máximos permitidos } \\
\text { (VMP) para consumo humano: }\end{array}$ & $\begin{array}{c}\text { Portaria do Ministério da Saúde } \\
\mathrm{n}^{\circ} 518(2004)\end{array}$ & $\begin{array}{c}\text { Portaria do Ministério da Saúde } \\
\mathrm{n}^{\circ} 2914(2011)\end{array}$ \\
\hline $\mathrm{pH}$ & 6 à 9,5 & 6 à 9,0 \\
\hline
\end{tabular}

Não especificado $\left(^{*}\right)$.

\subsection{Amostragem de Água}

Foram coletadas amostras para análises hidroquímicas em poços rasos e profundos em cada unidade geológica da região em estudo. Durante a coleta foi adicionado $10 \mathrm{ml}$ de ácido nítrico em um dos frascos da coleta para evitar, diminuir ou retardar alterações das características iniciais da amostra durante o transporte até o laboratório.

As análises hidroquímicas foram realizadas pelo Laboratório de Águas da Pontifícia Universidade Católica do Rio de Janeiro (LABAS/ PUC-RJ). Nestas análises foram verificados os seguintes parâmetros: Odor; Sólidos em Suspensão; Cor aparente; Turbidez; $\mathrm{pH}$; Condutividade a $25^{\circ} \mathrm{C}$; Dureza total; Sulfato $\left(\mathrm{SO}_{4}{ }^{-2}\right)$; Alcalinidade em $\mathrm{CaCO}_{3}$; Carbonato em $\mathrm{CO}_{3}{ }^{-2}$; Bicarbonato em $\mathrm{HCO}_{3}{ }^{-}$; Cloreto $\left(\mathrm{Cl}^{-}\right)$; Fosfato em $\mathrm{HPO}_{4}{ }^{-2}$; Antimônio (Sb); Arsênio (As); Bário (Ba); Cádmio (Cd); Cromo (Cr); Cobre (Cu); Nitrito $\left(\mathrm{NO}_{2}\right)$; Nitrato $\left(\mathrm{NO}_{3}\right)$; Cianeto $(\mathrm{CN})$; Chumbo $(\mathrm{Pb})$; Manganês $(\mathrm{Mn})$; Mercúrio(Hg); Selênio (Se); Sódio $(\mathrm{Na})$; Fluoreto( $\left.\mathrm{F}^{-}\right)$; Alumínio (Al); Cálcio (Ca); Ferro (Fe); Zinco (Zi); Berílio (Be); Cobalto (Co); Lítio (Li); Magnésio (Mg); Vanádio (V); Molibidênio (Mo); Potássio (K), entre outros.

\subsection{Análise dos dados}

Com a utilização do software AquaChem ${ }^{\circledR}$ 5.1, foi realizado o balanço iônico e elaborados os diagramas de Piper e de Stiff para fins de caracterização hidroquímica da área de estudo.

Utilizou-se os softwares ArcGis/ArcMap 9.4®, AutoCAD 2008 e Excel 2003 para confecção dos mapas para melhor visualização espacial dos resultados.

Considerando que as águas subterrâneas captadas serão usadas para consumo humano, foram

Persp. online: exatas \& eng., Campos dos Goytacazes, 3 (6), 25-41, 2013

seer.perspectivasonline.com.br 
observados os parâmetros e padrões de potabilidade da Portaria n 2914 do Ministério da Saúde (2011), Portaria ${ }^{\circ} 518$ do Ministério da Saúde (2004), Resolução nº 357 (2005) e a Resolução n 396 (2008).

\section{RESULTADOS E DISCUSSÕES}

\subsection{Cadastro de Pontos e Parâmetros Físicos Medidos em Campo}

Os resultados obtidos em laboratório das amostras de água coletadas apresentaram os seguintes parâmetros: físicos e físico-químicos (Tabela 4), dos ânions (Tabela 5), das substâncias controladas (Tabela 6) e dos elementos (Tabela 7).

Tabela 4 - Resultados de parâmetros físicos e físico-químicos obtidos em laboratório

\begin{tabular}{|c|c|c|c|c|c|c|c|c|c|}
\hline Poços & 1 & 2 & 3 & 4 & 5 & 6 & 7 & 8 & 9 \\
\hline $\begin{array}{l}\text { Aspecto } \\
\text { Natural* }\end{array}$ & \multicolumn{2}{|c|}{$\begin{array}{c}\text { Turva e } \\
\text { amarelada }\end{array}$} & \multicolumn{4}{|c|}{ Límpido e incolor } & $\begin{array}{l}\text { límpido e } \\
\text { amarelado }\end{array}$ & $\begin{array}{c}\text { turvo e } \\
\text { ferrugem }\end{array}$ & $\begin{array}{l}\text { Límpido e } \\
\text { incolor }\end{array}$ \\
\hline Odor a frio & \multicolumn{9}{|c|}{ Ausente } \\
\hline $\begin{array}{l}\text { Sólidos } \\
\text { suspensão* }\end{array}$ & \multicolumn{2}{|c|}{ Sim } & \multicolumn{2}{|c|}{ Não } & Sim & Não & \multicolumn{2}{|c|}{ Sim } & Não \\
\hline Cor aparente $(\mathrm{uH})$ & 154 & 16,7 & 4,6 & 1,7 & 5,6 & 1,7 & 80,6 & 500 & 1,6 \\
\hline Cor real $(\mathrm{uH}) *$ & 7,9 & 1,3 & 3,4 & 1,2 & 2,4 & 1,3 & 71,9 & 58,1 & 1,2 \\
\hline Turbidez (uT) & 118 & 14 & 1 & 1 & 3 & 1 & 5 & 164 & 1 \\
\hline $\mathrm{pH}$ a $25^{\circ} \mathrm{C}$ & 5,8 & 6,3 & 3,9 & 4,3 & 5,9 & 4,4 & 6,2 & 6,4 & 4,9 \\
\hline $\begin{array}{l}\text { Condutividade } \\
25^{\circ} \mathrm{C}(\mu \mathrm{s} / \mathrm{cm}) *\end{array}$ & 1100 & 732 & 4000 & 660 & 389 & 249 & 330 & 360 & 860 \\
\hline $\begin{array}{l}\text { Evaporação } \\
180^{\circ} \mathrm{C}(\mathrm{mg} / \mathrm{L}) *\end{array}$ & 736 & 474 & $\begin{array}{c}2,2 \times 10 \\
3\end{array}$ & 428 & 257 & 168 & 219 & 238 & 555 \\
\hline $\begin{array}{l}\text { Dureza total } \\
\mathrm{CaCO} 3(\mathrm{mg} / \mathrm{L})\end{array}$ & 160 & 250 & 443 & 68 & 49,5 & 45,2 & 111 & 73,1 & 56,6 \\
\hline $\begin{array}{l}\text { Dureza } \\
\text { permanente } \\
\mathrm{CaCO} 3(\mathrm{mg} / \mathrm{L}) *\end{array}$ & 153 & 153 & 443 & 68 & 48,1 & 45,2 & 61,4 & 41,6 & 51,1 \\
\hline $\begin{array}{l}\text { Dureza temporária } \\
\mathrm{CaCO} 3(\mathrm{mg} / \mathrm{L}) *\end{array}$ & 7,03 & 96,3 & 0 & - & 1,37 & - & 49,4 & 31,5 & 5,53 \\
\hline
\end{tabular}

\begin{tabular}{|c|c|c|c|c|c|}
\hline $\begin{array}{c}\text { V M P para consumo } \\
\text { humano: }\end{array}$ & $\begin{array}{c}\text { Odor } \\
\text { (intensidade) }\end{array}$ & $\begin{array}{c}\text { Cor Aparente } \\
(\mathrm{uH})\end{array}$ & $\begin{array}{c}\text { Turbidez } \\
(\mathrm{uT})\end{array}$ & $\mathrm{pH}$ & $\begin{array}{c}\text { Dureza Total } \\
(\mathrm{mg} / \mathrm{L})\end{array}$ \\
\hline $\begin{array}{c}\text { Portaria do M. S. } \mathrm{n}^{\circ} 518 \\
(2004)\end{array}$ & Não Objetável & 15 & 5 & 6 a 9,5 & 500 \\
\hline $\begin{array}{c}\text { Portaria do M. S. } \mathrm{n}^{\circ} 2914 \\
(2011)\end{array}$ & 6 & 15 & 5 & 6 a 9,0 & 500 \\
\hline
\end{tabular}

Não especificado $\left(^{*}\right)$. 
Tabela 5 - Resultados dos ânions obtidos em laboratório

\begin{tabular}{|l|c|c|c|c|c|c|c|c|c|}
\hline \multicolumn{1}{|c|}{ Poços } & 1 & 2 & 3 & 4 & 5 & 6 & 7 & 8 & 9 \\
\hline $\mathrm{SO}_{4}^{-2}(\mathrm{mg} / \mathrm{L})$ & 114 & 20,8 & 240 & 104 & 28 & 48 & 28,1 & 25,7 & 16,3 \\
\hline Alcalinidade em $\mathrm{CaCO}_{3}(\mathrm{mg} / \mathrm{L})$ & 7,03 & 96,3 & 0 & 0 & 1,37 & 0 & 49,4 & 31,5 & 5,53 \\
\hline Carbonato em $\mathrm{CO}_{3}^{-2}(\mathrm{mg} / \mathrm{L}) *$ & 0 & 0 & 0 & 0 & 0 & 0 & 0 & 0 & 0 \\
\hline${\text { Bicarbonato em } \mathrm{HCO}_{3}^{-}(\mathrm{mg} / \mathrm{L})}^{*}$ & 8,58 & 118 & 0 & 0 & 1,67 & 0 & 60,3 & 38,4 & 6,74 \\
\hline $\mathrm{Br}^{-}(\mathrm{mg} / \mathrm{L}) *$ & 0,87 & 0,47 & $<1$ & 0,45 & 0,63 & 0,32 & 0,25 & 0,25 & 0,74 \\
\hline $\mathrm{Cl}^{-}(\mathrm{mg} / \mathrm{L})$ & 281 & 171 & 979 & 114 & 85,7 & 25,5 & 11,4 & 64,9 & 235 \\
\hline${\text { Fosfato em } \mathrm{HPO}_{4}{ }^{-2}(\mathrm{mg} / \mathrm{L})}^{*}$ & 0,096 & $<0,01$ & $<1$ & 0,2 & $<0,1$ & 0,23 & $<0,1$ & 3,29 & 0,37 \\
\hline $\mathrm{I}^{-}(\mathrm{mg} / \mathrm{L}) *$ & \multicolumn{7}{|c|}{ Não determinado } \\
\hline
\end{tabular}

\begin{tabular}{|c|c|c|c|}
\hline V M P para consumo humano: & $\begin{array}{c}\text { Sulfato }\left(\mathrm{SO}_{4}^{-2}\right) \\
(\mathrm{mg} / \mathrm{L})\end{array}$ & $\begin{array}{c}\text { Alcalinidade em } \\
\mathrm{CaCO}_{3}(\mathrm{uH})\end{array}$ & $\begin{array}{c}\text { Cloreto (Cl-) } \\
(\mathrm{mg} / \mathrm{L})\end{array}$ \\
\hline Portaria do M. S. $n^{\circ} 518(2004)$ & 250 & 500 & 250 \\
\hline Portaria do M. S. $\mathrm{n}^{\circ} 2914(2011)$ & 250 & 15 & 250 \\
\hline
\end{tabular}

Não especificado $\left(^{*}\right)$.

Tabela 6 - Resultados das substâncias controladas obtidas em laboratório

\begin{tabular}{|l|c|c|c|c|c|c|c|c|c|}
\hline \multicolumn{1}{|c|}{ Poços } & 1 & 2 & 3 & 4 & 5 & 6 & 7 & 8 & 9 \\
\hline $\mathrm{Sb}(\mathrm{mg} / \mathrm{L})$ & 0,002 & $<0,001$ & $<0,001$ & $<0,001$ & $<0,001$ & $<0,001$ & $<0,001$ & 0,002 & $<0,001$ \\
\hline $\mathrm{As}(\mathrm{mg} / \mathrm{L})$ & $<0,01$ & $<0,01$ & $<0,01$ & $<0,01$ & $<0,01$ & $<0,01$ & $<0,01$ & $<0,01$ & $<0,01$ \\
\hline $\mathrm{Ba}(\mathrm{mg} / \mathrm{L})$ & 0,027 & 0,17 & 0,053 & 0,021 & 0,025 & 0,045 & 0,033 & 0,076 & 0,33 \\
\hline $\mathrm{B}(\mathrm{mg} / \mathrm{L})$ & $<0,5$ & $<0,5$ & $<0,5$ & $<0,5$ & $<0,5$ & $<0,5$ & $<0,5$ & $<0,5$ & $<0,5$ \\
\hline $\mathrm{Cd}(\mathrm{mg} / \mathrm{L})$ & $<0,001$ & $<0,001$ & $<0,001$ & $<0,001$ & $<0,001$ & 0,002 & $<0,001$ & $<0,001$ & $<0,001$ \\
\hline $\mathrm{Cr}(\mathrm{mg} / \mathrm{L})$ & 0,003 & 0,004 & 0,001 & 0,003 & 0,002 & $<0,001$ & 0,001 & 0,004 & 0,006 \\
\hline $\mathrm{Cu}(\mathrm{mg} / \mathrm{L})$ & 0,03 & 0,003 & 0,005 & 0,003 & $<0,001$ & 0,001 & 0,005 & 0,022 & 0,001 \\
\hline $\mathrm{NO}_{2}(\mathrm{mg} / \mathrm{L})$ & 0,36 & 0,063 & \multicolumn{7}{|c|}{ Não determinado } \\
\hline $\mathrm{NO}_{3}(\mathrm{mg} / \mathrm{L})$ & 27,6 & 0,37 & 145 & 4,86 & 4,95 & 11,8 & 2,23 & 4,22 & 6,19 \\
\hline $\mathrm{CN}(\mathrm{mg} / \mathrm{L})$ & \multicolumn{7}{|c|}{$\mathrm{Na})$} & \multicolumn{7}{|c|}{ determinado } \\
\hline $\mathrm{Pb}(\mathrm{mg} / \mathrm{L})$ & 0,015 & $<0,001$ & 0,005 & 0,001 & $<0,001$ & $<0,001$ & $<0,001$ & 0,002 & 0,003 \\
\hline $\mathrm{Mn}(\mathrm{mg} / \mathrm{L})$ & 0,36 & 0,36 & 0,076 & 0,03 & 0,087 & 0,04 & 0,014 & 0,14 & 0,41 \\
\hline $\mathrm{Hg}(\mathrm{mg} / \mathrm{L})$ & $<0,001$ & $<0,001$ & 0,002 & $<0,001$ & $<0,001$ & $<0,001$ & $<0,001$ & $<0,001$ & $<0,001$ \\
\hline $\mathrm{Ni}(\mathrm{mg} / \mathrm{L})$ & 0,004 & 0,002 & 0,008 & 0,001 & $<0,001$ & 0,001 & 0,002 & 0,005 & 0,003 \\
\hline $\mathrm{Se}(\mathrm{mg} / \mathrm{L})$ & 0,003 & 0,002 & 0,019 & 0,002 & 0,003 & 0,001 & $<0,001$ & 0,002 & 0,004 \\
\hline $\mathrm{Na}(\mathrm{mg} / \mathrm{L})$ & 179 & 58,6 & 483 & 76,7 & 44,7 & 15,1 & 13,8 & 33 & 108 \\
\hline $\mathrm{F}^{-}(\mathrm{mg} / \mathrm{L})$ & 0,028 & 0,06 & $<1$ & $<0,1$ & $<0,1$ & $<0,1$ & 0,17 & $<0,1$ & $<0,2$ \\
\hline
\end{tabular}




\begin{tabular}{|c|c|c|}
\hline V M P para consumo humano: & Portaria do M. S. n 518 (2004) & Portaria do M. S. n 2914 (2011) \\
\hline Antimônio (Sb) (mg/L) & 0,005 & * \\
\hline Arsênio (As) (mg/L) & 0,01 & * \\
\hline Bário (Ba) (mg/L) & 0,7 & $*$ \\
\hline Cádmio $(\mathrm{Cd})(\mathrm{mg} / \mathrm{L})$ & 0,005 & * \\
\hline Cromo $(\mathrm{Cr})(\mathrm{mg} / \mathrm{L})$ & 0,05 & * \\
\hline Cobre $(\mathrm{Cu})(\mathrm{mg} / \mathrm{L})$ & 2 & $*$ \\
\hline V M P para consumo humano: & Portaria do M. S. n 518 (2004) & Portaria do M. S. n 2914 (2011) \\
\hline Nitrito $\left(\mathrm{NO}_{2}\right)(\mathrm{mg} / \mathrm{L})$ & 1 & * \\
\hline Nitrato $\left(\mathrm{NO}_{3}\right)(\mathrm{mg} / \mathrm{L})$ & 10 & $*$ \\
\hline Cianeto $(\mathrm{CN})(\mathrm{mg} / \mathrm{L})$ & 0,07 & $*$ \\
\hline Chumbo $(\mathrm{Pb})(\mathrm{mg} / \mathrm{L})$ & 0,01 & $*$ \\
\hline Manganês (Mn) (mg/L) & 0,1 & 0,1 \\
\hline Mercúrio $(\mathrm{Hg})(\mathrm{mg} / \mathrm{L})$ & 0,001 & * \\
\hline Selênio $(\mathrm{Se})(\mathrm{mg} / \mathrm{L})$ & 0,01 & $*$ \\
\hline Sódio $(\mathrm{Na})(\mathrm{mg} / \mathrm{L})$ & 200 & 200 \\
\hline Fluoreto(F-) $(\mathrm{mg} / \mathrm{L})$ & 1,5 & $*$ \\
\hline
\end{tabular}

Não especificado (*).

\begin{tabular}{|c|c|c|}
\hline V M P para consumo humano: & $\begin{array}{c}\text { Resolução do CONAMA n }{ }^{\circ} 357 \\
(2005)\end{array}$ & $\begin{array}{c}\text { Portaria do M. S. } \mathrm{n}^{\circ} 2914 \\
(2011)\end{array}$ \\
\hline Boro $(\mathrm{B})(\mathrm{mg} / \mathrm{L})$ & 0,5 & ${ }^{*}$ \\
\hline Níquel $(\mathrm{Ni})(\mathrm{mg} / \mathrm{L})$ & 0,0025 & ${ }^{*}$ \\
\hline
\end{tabular}

Não especificado (*). 
Tabela 7 - Resultados dos elementos químicos obtidos em laboratório

\begin{tabular}{|l|c|c|c|c|c|c|c|c|c|}
\hline \multicolumn{1}{|c|}{ Poços } & 1 & 2 & 3 & 4 & 5 & 6 & 7 & 8 & 9 \\
\hline $\mathrm{Al}(\mathrm{mg} / \mathrm{L})$ & 0,31 & 0,021 & 5,75 & 0,75 & 0,083 & 0,4 & 0,17 & 2,01 & 0,64 \\
\hline $\mathrm{Be}(\mathrm{mg} / \mathrm{L})$ & $<0,001$ & $<0,001$ & $<0,001$ & $<0,001$ & $<0,001$ & $<0,001$ & $<0,001$ & $<0,001$ & $<0,001$ \\
\hline $\mathrm{Ca}(\mathrm{mg} / \mathrm{L}) *$ & 28,6 & 64 & 68,7 & 6,1 & 7,67 & 4,63 & 42,7 & 21,5 & 4,82 \\
\hline $\mathrm{Co}(\mathrm{mg} / \mathrm{L})$ & 0,003 & $<0,001$ & 0,005 & 0,002 & $<0,001$ & 0,001 & $<0,001$ & 0,002 & 0,01 \\
\hline $\mathrm{Sc}(\mathrm{mg} / \mathrm{L}) *$ & $<0,001$ & 0,0031 & 0,01 & 0,003 & $<0,001$ & 0,001 & $<0,001$ & 0,003 & 0,002 \\
\hline $\mathrm{Sn}(\mathrm{mg} / \mathrm{L}) *$ & $<0,001$ & $<0,001$ & $<0,0001$ & $<0,001$ & $<0,001$ & $<0,001$ & $<0,001$ & 0,003 & $<0,001$ \\
\hline $\mathrm{Sr}(\mathrm{mg} / \mathrm{L})$ & 0,17 & 0,5 & 0,71 & 0,089 & 0,058 & 0,06 & 0,21 & 0,18 & 0,063 \\
\hline $\mathrm{Fe}(\mathrm{mg} / \mathrm{L})$ & 3,04 & 9,84 & 0,68 & 0,032 & 1,02 & 0,01 & 2,06 & 1,05 & 0,042 \\
\hline $\mathrm{Li}(\mathrm{mg} / \mathrm{L})$ & $<0,01$ & 0,014 & $<0,01$ & $<0,01$ & $<0,01$ & $<0,01$ & $<0,01$ & 0,013 & 0,003 \\
\hline $\mathrm{Mg}(\mathrm{mg} / \mathrm{L}) *$ & 21,6 & 21,8 & 65,9 & 12,8 & 7,38 & 8,18 & 1,07 & 4,75 & 10,8 \\
\hline $\mathrm{Mo}(\mathrm{mg} / \mathrm{L})$ & $<0,001$ & $<0,001$ & $<0,001$ & $<0,001$ & $<0,001$ & $<0,001$ & 0,002 & 0,002 & $<0,001$ \\
\hline $\mathrm{K}(\mathrm{mg} / \mathrm{L}) *$ & 10,9 & 9,3 & 114 & 8,12 & 3,02 & 8,52 & 11,3 & 10,2 & 9,49 \\
\hline $\mathrm{SiO}(\mathrm{mg} / \mathrm{L}) *$ & 4,58 & 77,3 & 9,33 & 8,51 & 4,7 & 17,3 & 7,93 & 50,8 & 9,53 \\
\hline $\mathrm{Ti}(\mathrm{mg} / \mathrm{L}) *$ & 0,003 & 0,002 & 0,012 & $<0,001$ & $<0,001$ & 0,001 & 0,001 & 0,008 & 0,004 \\
\hline $\mathrm{W}(\mathrm{mg} / \mathrm{L}) *$ & $<0,001$ & $<0,001$ & $<0,001$ & $<0,001$ & $<0,001$ & $<0,001$ & $<0,001$ & $<0,001$ & $<0,001$ \\
\hline $\mathrm{V}(\mathrm{mg} / \mathrm{L})$ & $<0,001$ & $<0,001$ & 0,005 & $<0,001$ & $<0,001$ & $<0,001$ & $<0,001$ & 0,018 & 0,001 \\
\hline $\mathrm{Zn} \mathrm{(mg/L)}$ & 0,13 & 0,045 & 0,051 & 0,033 & 0,033 & 0,023 & 0,061 & 0,16 & 0,024 \\
\hline
\end{tabular}

\begin{tabular}{|c|c|c|c|}
\hline V M P para consumo humano: & Alumínio (Al) & Ferro (Fe) & Zinco (Zi) \\
\hline Portaria do M. S. $n^{\circ} 518(2004)$ & 0,2 & 0,3 & 5 \\
\hline Portaria do M. S. $n^{\circ} 2914(2011)$ & 250 & 15 & 250 \\
\hline
\end{tabular}

\begin{tabular}{|c|c|c|}
\hline V M P para consumo humano: & $\begin{array}{c}\text { Resolução do CONAMA n }{ }^{\circ} 357 \\
(2005)\end{array}$ & $\begin{array}{c}\text { Portaria do M. S. } \mathrm{n}^{\circ} 2914 \\
(2011)\end{array}$ \\
\hline Berílio $(\mathrm{Be})(\mathrm{mg} / \mathrm{L})$ & 0,04 & ${ }^{*}$ \\
\hline Cobalto $(\mathrm{Co})(\mathrm{mg} / \mathrm{L})$ & 0,05 & ${ }^{*}$ \\
\hline Lítio $(\mathrm{Li})(\mathrm{mg} / \mathrm{L})$ & 2,5 & ${ }^{*}$ \\
\hline Vanádio $(\mathrm{V})(\mathrm{mg} / \mathrm{L})$ & 0,1 & ${ }^{*}$ \\
\hline
\end{tabular}

Não especificado $\left(^{*}\right)$.

\begin{tabular}{|c|c|c|}
\hline V M P para consumo humano: & $\begin{array}{c}\text { Resolução do CONAMA n }{ }^{\circ} 396 \\
(2008)\end{array}$ & $\begin{array}{c}\text { Portaria do M. S. } \mathrm{n}^{\circ} 2914 \\
(2011)\end{array}$ \\
\hline Molibidênio $(\mathrm{Mo})(\mathrm{mg} / \mathrm{L})$ & 0,007 & $*$ \\
\hline
\end{tabular}

Não especificado (*). 
De acordo com os resultados das nove amostras obtidos em laboratório pode-se observar que os seguintes elementos encontram-se fora do padrão da Portaria do Ministério da Saúde n 2914 (2011), Portaria do Ministério da Saúde no 518 (2004), da Resolução do CONAMA n 357 (2005) e Resolução do CONAMA n 396 (2008): Chumbo, Mercúrio, Selênio e Sódio em 11,11\% das amostras; Cloreto em 22,22\%; Nitrato em 33,33\%; Manganês e Níquel em 44,44\%; Alumínio em 77,77\% e Ferro em 88,88\% das amostras.

\subsection{Diagramas de Piper e de Stiff}

O estudo e visualização dos resultados de análises hidroquímicas podem ser simplificados com a utilização de gráficos e diagramas, como os Diagramas Triangular de Piper e de Stiff (Figuras 4 e 5), que servem para classificar e comparar os distintos grupos de água quanto aos íons dominantes (Tabela 8) expressos em miliequivalente (Equação 1), conforme fórmula abaixo.

Miliequivalente $\left(\frac{m e q}{L}\right)=\frac{\text { Miligramas / litro }(\mathrm{mg} / \mathrm{L})}{\operatorname{PesoAtomico}(\mathrm{Mol})} \times$ ValênciaElemento

(Equação 1)

Tabela 8- Elementos utilizados na confecção dos diagramas de Piper e de Stiff

\begin{tabular}{|l|c|c|c|c|c|c|c|}
\hline Poços & $\begin{array}{c}\mathrm{SO}_{4}^{-2} \\
\left(\mathrm{~m}_{\mathrm{eq}} / \mathrm{L}\right)\end{array}$ & $\begin{array}{c}\text { Bicarbonato em } \mathrm{HCO}^{-3} \\
\left(\mathrm{~m}_{\mathrm{eq}} / \mathrm{L}\right)\end{array}$ & $\begin{array}{c}\mathrm{Cl}^{-} \\
\left(\mathrm{m}_{\mathrm{eq}} / \mathrm{L}\right)\end{array}$ & $\begin{array}{c}\mathrm{Ca} \\
\left(\mathrm{m}_{\mathrm{eq}} / \mathrm{L}\right)\end{array}$ & $\begin{array}{c}\mathrm{Mg} \\
\left(\mathrm{m}_{\mathrm{eq}} / \mathrm{L}\right)\end{array}$ & $\begin{array}{c}\mathrm{Na} \\
\left(\mathrm{m}_{\mathrm{eq}} / \mathrm{L}\right)\end{array}$ & $\begin{array}{c}\mathrm{K} \\
\left(\mathrm{m}_{\mathrm{eq}} / \mathrm{L}\right)\end{array}$ \\
\hline 1 & 2,375 & 0,141 & 7,915 & 1,430 & 1,778 & 7,783 & 0,279 \\
\hline 2 & 0,433 & 1,934 & 4,817 & 3,200 & 1,794 & 2,548 & 0,238 \\
\hline 3 & 5,000 & 0,000 & 27,577 & 3,435 & 5,424 & 21,000 & 2,923 \\
\hline 4 & 2,167 & 0,000 & 3,211 & 0,305 & 1,053 & 3,335 & 0,208 \\
\hline 5 & 0,583 & 0,027 & 2,414 & 0,384 & 0,607 & 1,943 & 0,077 \\
\hline 6 & 1,000 & 0,000 & 0,718 & 0,232 & 0,673 & 0,657 & 0,218 \\
\hline 7 & 0,585 & 0,989 & 0,321 & 2,135 & 0,088 & 0,600 & 0,290 \\
\hline 8 & 0,535 & 0,630 & 1,828 & 1,075 & 0,391 & 1,435 & 0,262 \\
\hline 9 & 0,340 & 0,110 & 6,620 & 0,241 & 0,889 & 4,696 & 0,243 \\
\hline
\end{tabular}

Persp. online: exatas \& eng., Campos dos Goytacazes, 3 (6), 25-41, 2013 


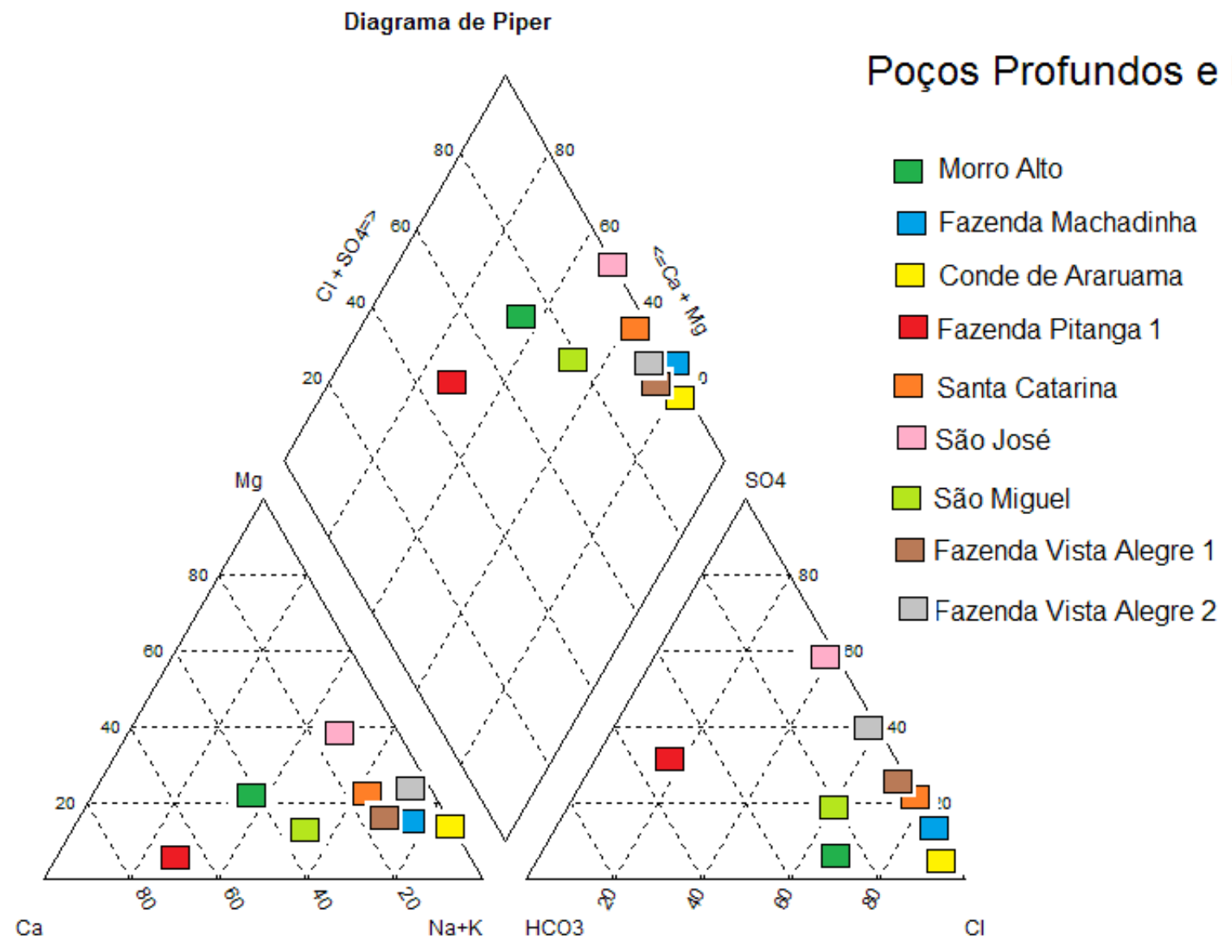

\section{Poços Profundos e Rasos}

Morro Alto

Fazenda Machadinha

Conde de Araruama

azenda Pitanga 1

Santa Catarina

São José

São Miguel

Alegre 1

Figura 4 - Diagrama de Piper referente aos poços profundos e rasos

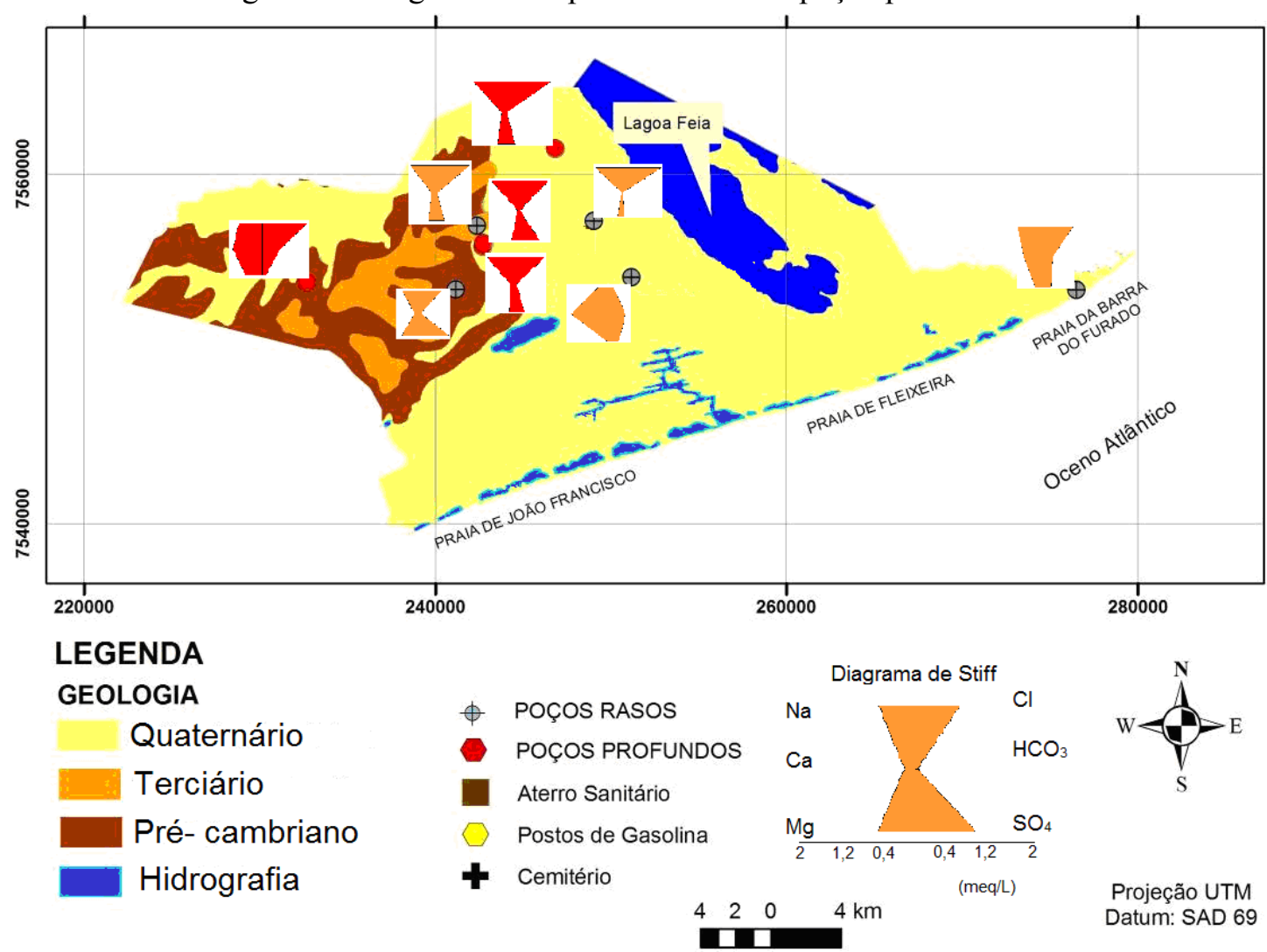

Figura 5 - Mapa de espacialização dos diagramas de Stiff (Fonte: Oficina de geologia e geoprocessamento OFIGEO/UENF. Compilado do CPRM, 2001)

Persp. online: exatas \& eng., Campos dos Goytacazes, 3 (6), 25-41, 2013 
De acordo com os diagramas de Piper e de Stiff os poços profundos da Fazenda Machadinha (poço profundo 3) e Fazenda Vista Alegre 1 (poço profundo 1) são classificados como Águas Cloretadas Sódicas, enquanto o poço profundo de Morro Alto (poço profundo 2) como Água Cloretada Cálcica Sódica e Fazenda Vista Alegre 2 (poço profundo 4) como Cloretada Sulfatada Sódica.

Os poços rasos de Conde de Ararruama (poço raso 9) e Santa Catarina (poço raso 5) são classificados como Águas Cloretadas Sódicas, enquanto o da Fazenda Pitanga (poço raso 7) como Água Bicarbonatada Sulfatada Cálcica, o de São Miguel (poço raso 8) como Cloretada Sódica Cálcica e o de São José (poço raso 6) como Água Sulfatada Sódica Magnesiana.

\section{CONCLUSÕES}

Em conformidade com o que foi exposto neste trabalho, com os resultados obtidos com as análises hidroquímicas das águas subterrâneas em laboratório e com os gráficos de Piper e de Stiff pode-se concluir que: os poços profundos e rasos de Quissamã possuem predominância em sua classificação de Águas Cloretadas Sódicas.

Nas águas subterrâneas foram medidas em campo 09 amostras de poços rasos e profundos em que a condutividade elétrica variou de 146 a $3240 \mu \mathrm{S} / \mathrm{cm}$ (em campo) e 249 a $4000 \mu \mathrm{S} / \mathrm{cm}$ (em laboratório); o pH variou de 3,6 a 7,0 (em campo) e 3,9 a 6,2 (em laboratório). Com os resultados de condutividade encontrados pode-se notar uma amostra de poço profundo 3 (Fazenda Machadinha) com valor superior a $1000 \mu \mathrm{S} / \mathrm{cm}$, indicando maior presença de sais como o sódio e o cloreto. Segundo a Portaria do Ministério da Saúde ${ }^{\circ}$ 518 e 2914 o pH permitido para consumo humano deve variar entre 6 e 9,0, entretanto todas as amostras de poços rasos e duas de poços profundos medidos apresentaram o valor do $\mathrm{pH}$ abaixo do mínimo permitido, ou seja, indicando acidez.

Em 77,77\% e 88,88\% das amostras foram encontradas concentrações de Alumínio e Ferro bem acima dos valores permitidos pela Portaria do Ministério da Saúde ${ }^{\circ} 518$ (2004) e da Portaria do Ministério da Saúde $n^{\circ} 2914$ (2011). O acúmulo de alumínio no corpo pode danificar o sistema nervoso central, a estrutura óssea do ser humano e até causar câncer, já o ferro em sua carência causa anemia e seu excesso aumenta a incidência de problemas cardíacos e diabetes (Teixeira, 1998). Estes elementos são facilmente removidos da água através de filtragem com filtro de cerâmico caseiro.

Ainda, foram encontradas amostras com elementos fora dos padrões das portarias e resoluções adotadas, como o cloreto, nitrato, chumbo, manganês, mercúrio, selênio, sódio e níquel, mas em menor número de casos.

A água subterrânea quando captada de forma adequada apresenta baixo custo de tratamento, no caso da região será necessário tratamento, pois elementos foram encontrados fora do padrão, sendo alguns bem nocivos à saúde da população, que consome essas águas sem o conhecimento do risco que estão expostos.

Para todo município, recomendam-se trabalhos de educação ambiental com a finalidade de conscientização da comunidade sobre a importância da água subterrânea como bem de consumo sendo instruídas ações práticas como a utilização de cisternas, filtros, construção de fossas longe de poços, dentre outros.

Persp. online: exatas \& eng., Campos dos Goytacazes, 3 (6), 25-41, 2013

seer.perspectivasonline.com.br 


\section{REFERÊNCIAS}

BRASIL. Ministério da Saúde. Portaria nº 518. Dispõe sobre o padrão de qualidade das águas. Brasília (DF), 2004.

BRASIL. Ministério da Saúde. Portaria no 2914. Dispõe sobre o padrão de qualidade das águas. Brasília (DF), 2012.

CAPUCCI, E., MARTINS, A. M., MANSUR, K.L., MONSORES, A.L.M. Poços tubulares e outras captações de água subterrânea - Orientação aos usuários - Rio de Janeiro- RJ, 2001.

CONAMA - CONSELHO NACIONAL DO MEIO AMBIENTE. Resolução n 35723 de janeiro de 2005. Disponível em: www.mma.gov.br/port/conama. Acesso em 05 de janeiro de 2012.

CONAMA - CONSELHO NACIONAL DO MEIO AMBIENTE. Resolução nº 39603 de abril de 2008. Disponível em: www.mma.gov.br/port/conama. Acesso em 05 de janeiro de 2012.

CPRM - Companhia de Pesquisa e Recursos Minerais. Ministério das Minas e Energia / Secretaria de Minas e Metalurgia / Companhia de Pesquisa de Recursos Minerais. Projeto Rio de Janeiro, CD-ROM. Brasília, 2001.

CUSTÓDIO, E.; LAMAS, M. R.; Hidrogeologia Subterrânea. Barcelona, Editora Omega. 2359 p., 1983.

FETTER, C. W. Applied hydrogeology. New Jersey, Prentice Hall, 2001.

FREEZE, R. A.; CHERRY, J. A. Groundwater. Prentece Hall, Inc. Engewood Cliffs, 1979.

IBGE - Instituto Brasileiro de Geografia e Estatística, http://www.ibge.com.br em 2010. Acesso em 02/09/2010.

OMS. Índices de Calidad de Água e Salud. Organizacion Mundial de la Salud. Disponível em $\leq$ http://www.who.int/water_sanitation_health/monitoring/2000 globs6.pdf $>$ Acesso em: 10 abril 2010. 1970.

OMS. Guidelines for Drinking-water Quality. Vol. 1, $3^{\mathrm{a}}$ ed. Organização Mundial de Saúde. Disponível em: <http://www.who.int/water_sanitation_health/dwq/gdwq3/en>. Acesso em: 23 outubro 2010. 2004.

SANTOS, A. C. Noções de Hidroquímica. In: Hidrogeologia: Conceitos e Aplicações. Coordenadores: Fernando Antônio Carneiro Feitosa / João Manoel Filho. Fortaleza. CPRM / LABHID - UFPE. 1ª Ed., 1997.

SANTOS, A. C. Noções de Hidroquímica. In: Hidrogeologia - Conceitos e Aplicações, 2000.

TEIXEIRA, F.H. Estudo comparativo para metais em ambientes aquático (Bacia Hidrográfica do Rio Guandu - RJ). 123p. Dissertação (Mestrado em Ciências). Programa de Pós-graduação de Engenharia Sanitária e Saúde Ambiental. FIOCRUZ/ENSP. Rio de Janeiro, 1998.

TOOD, D. K. Hidrologia águas subterrâneas. Rio de Janeiro: Editora Edgard Blücher Ltda., 1967.

Persp. online: exatas \& eng., Campos dos Goytacazes, 3 (6), 25-41, 2013

seer.perspectivasonline.com.br 
UNESCO. A. Groundwater Resources Sustainability Indicators IHP. VI Series on Groundwater No 14 Published in 2007 by the United Nations Educational, Scientific and Cultural Organization 7, Place de Fontenoy, 75352 Paris 07 SP (France) Composed by Marina Rubio, 93200 Saint-Denis, 2007.

USEPA. U.S. Environmental Protection Agency. Disponível em $<$ www.epa.gov/history/index.htm $>$ Acesso em 10 junho 2010. 1975. 